\title{
ON THE SPECTRAL THEORY OF SYMMETRIC FINITE OPERATORS $\left({ }^{1}\right)$
}

\author{
BY \\ HARRO HEUSER
}

Let $A$ be a linear operator defined on a linear system $X$ and let $N(A-\lambda I)$ be the null space, $R(A-\lambda I)$ the range of $A-\lambda I$, and $\lambda$ an arbitrary complex number. We call $A$ a finite operator if for each $\lambda \neq 0$ the dimensions of $N(A-\lambda I)$ and $X / R(A-\lambda I)$ are finite and equal. The present paper is concerned with an iteration method for determining characteristic values and characteristic elements of symmetric finite operators on a not necessarily complete Hilbert space $X$ and with the structure of the spectrum of such operators. The following two theorems are the basis of our exposition.

THEOREM 1. If $A$ is a symmetric finite operator on $X$ and $C \sigma(A)$ its continuous spectrum, then $C \sigma(A)-\{0\}$ consists of all the limit points of characteristic values of $A$ which are different from zero and no characteristic values themselves $\left({ }^{2}\right)$.

Theorem 2. If $A$ is a symmetric finite operator on $X$ and $A \neq 0$, then $A$ has a characteristic value different from zero and each element $A x$ can be expanded in a series

$$
A x=\sum_{e \in E}(A x, e) e=\sum_{e \in E} \lambda(x, e) e,
$$

where $E$ is a complete orthonormal system of characteristic elements of $A$ corresponding to the characteristic values different from zero $\left({ }^{3}\right)$.

Theorem 2 gives rise to a convenient definition. We say that a number $\lambda \neq 0$ contributes to the element $x$ if $\lambda$ actually appears in the series (1). It is readily seen that this definition does not depend on the particular system, $E$, chosen.

In the following, it will be convenient to suppose that $A$ is not only symmetric and finite, but also bounded and positive. Excluding the trivial case $A=0$, we further assume throughout $A \neq 0$. Under these assumptions, the

Received by the editors February 5, 1959.

(1) The research reported in this article was done in the Range Instrumentation Development Division, White Sands Missile Range, New Mexico.

(2) A proof of this theorem can be found in [1]. The results of this thesis will be published in a forthcoming paper in the Mathematische Zeitschrift.

(3) This theorem was first proved by Professor $\mathrm{H}$. Wielandt in a lecture given at the University of Tübingen in the summer of 1952 . Another proof can be found in [1]. It is understood that the series (1) contains only those terms for which $(x, e) \neq 0$. The number of those terms is at most enumerable. 
set of characteristic values $\lambda \neq 0$ of $A$ is a nonempty bounded set of positive numbers. At the end of this paper it will be shown how to eliminate the hypothesis $A \geqq 0$.

After these preliminary remarks we can prove the following theorem:

Theorem 3. Let $x$ be an element of $X$ with $A x \neq 0$. Then at least one characteristic value of $A$ contributes to $x$, and $\lim _{k \rightarrow \infty}\left(\left\|A^{k} x\right\| /\left\|A^{k-1} x\right\|\right)$ exists and is equal to the least upper bound of all characteristic values contributing to $x$.

Proof. The first assertion follows directly from Theorem 2. Now let $e_{1}, e_{2}, \cdots$ be the sequence of elements of $E$ for which $(x, e) \neq 0, \lambda_{1}, \lambda_{2}, \cdots$ the sequence of the corresponding characteristic values, and let $\mu$ be the least upper bound of these $\lambda_{i}$. Consider the series

$$
\sum_{i=1}^{\infty} \sum_{k=1}^{\infty}\left(\zeta \lambda_{i}\right)^{k}\left|\left(x, e_{i}\right)\right|^{2}
$$

If $|\zeta|<1 / \mu$, then the series $\left.\sum_{k=1}^{\infty}\left|\zeta \lambda_{i}\right|^{k}\left(x, e_{i}\right)\right|^{2}$ is obviously convergent and has the sum $\left(\left|\zeta \lambda_{i}\right| /\left(1-\left|\zeta \lambda_{i}\right|\right)\right)\left|\left(x, e_{i}\right)\right|^{2}$. Since $\left|\zeta \lambda_{i}\right| /\left(1-\left|\zeta \lambda_{i}\right|\right)$ $\leqq|\zeta| \mu /(1-|\zeta| \mu)$, and since the series $\sum_{i=1}^{\infty}\left|\left(x, e_{i}\right)\right|^{2}$ converges by virtue of Bessel's inequality, it follows that the series $\sum_{i=1}^{\infty}\left(\left|\zeta \lambda_{i}\right| /\left(1-\left|\zeta \lambda_{i}\right|\right)\right)\left|\left(x, e_{i}\right)\right|^{2}$ is also convergent. Hence, by Cauchy's theorem, the rearranged series

$$
\sum_{k=1}^{\infty} \sum_{i=1}^{\infty}\left(\zeta \lambda_{i}\right)^{k}\left|\left(x, e_{i}\right)\right|^{2}
$$

converges in the circle $|\zeta|<1 / \mu$. Since by Theorem 1

$$
A^{k} x=\sum_{i=1}^{\infty} \lambda_{i}^{k}\left(x, e_{i}\right) e_{i}
$$

and therefore

$$
\left(A^{k} x, x\right)=\sum_{i=1}^{\infty} \lambda_{i}^{k}\left|\left(x, e_{i}\right)\right|^{2},
$$

we see that the power series

$$
\sum_{k=1}^{\infty}\left(A^{k} x, x\right) \zeta^{k}
$$

converges in $|\zeta|<1 / \mu$. On the other hand, this series cannot converge for any value of $\zeta$ with $|\zeta|>1 / \mu$, since otherwise all the series $\sum_{k=1}^{\infty}\left(\zeta \lambda_{i}\right)^{k}$ would be convergent by the same type of argument used above, which contradicts the fact that there is a $\lambda_{i}$ with $\left|\zeta \lambda_{i}\right|>1$. Therefore the radius of convergence of the power series (3) equals $1 / \mu$ from which we infer that $\mu=\lim \sup _{k \rightarrow \infty}\left(A^{k} x, x\right)^{1 / k}$. Now by the generalized Schwarz inequality [3, p. 260] 
1960] ON THE SPECTRAL THEORY OF SYMMETRIC FINITE OPERATORS 329

$$
\left(A^{k} x, x\right)^{2}=\left(A^{k-1} x, A x\right)^{2} \leqq\left(A^{k-1} x, x\right)\left(A^{k-1} A x, A x\right)=\left(A^{k-1} x, x\right)\left(A^{k+1} x, x\right)
$$

and therefore

$$
\frac{\left(A^{k} x, x\right)}{\left(A^{k-1} x, x\right)} \leqq \frac{\left(A^{k+1} x, x\right)}{\left(A^{k} x, x\right)}
$$

from which it follows that the sequence $\left(A^{k} x, x\right) /\left(A^{k-1} x, x\right)$ converges. This implies convergence of the sequence $\left(A^{k} x, x\right)^{1 / k}$, so that

$$
\mu=\lim _{k \rightarrow \infty}\left(A^{k} x, x\right)^{1 / k} \text {. }
$$

It follows that

$$
\mu=\lim _{k \rightarrow \infty}\left(A^{2 k} x, x\right)^{1 / 2 k}=\lim _{k \rightarrow \infty}\left(A^{k} x, A^{k} x\right)^{1 / 2 k}=\lim _{k \rightarrow \infty}\left(\left\|A^{k} x\right\|\right)^{1 / k}
$$

and since the sequence $\left\|A^{k} x\right\| /\left\|A^{k-1} x\right\|$ converges [3, p. 238], $\mu$ must equal $\lim _{k \rightarrow \infty}\left(\left\|A^{k} x\right\| /\left\|A^{k-1} x\right\|\right)$, which completes the proof.

Theorem 3 does not tell us whether or not $\mu$ contributes to $x$. The next theorem will close this gap.

TheOREM 4. Let $A x$ be different from zero and let

$$
\mu=\lim _{k \rightarrow \infty} \frac{\left\|A^{k} x\right\|}{\left\|A^{k-1} x\right\|} .
$$

Then

$$
\begin{aligned}
& \lim _{x \rightarrow \infty} \frac{\left\|A^{k} x\right\|}{\lambda^{k}}=0 \quad \text { for } \lambda>\mu ; \\
& \lim _{k \rightarrow \infty} \frac{\left\|A^{k} x\right\|}{\mu^{k}}=0, \text { when } \mu \text { does not contribute to } x ; \\
& \lim _{k \rightarrow \infty} \frac{\left\|A^{k} x\right\|}{\mu^{k}}=\rho \neq 0, \text { when } \mu \text { contributes to } x ; \rho
\end{aligned}
$$

equals the length of the projection of $x$ on $N(A-\mu I)$ along $R(A-\mu I)$;

$$
\lim _{\boldsymbol{k} \rightarrow \infty} \frac{\left\|A^{k} x\right\|}{\lambda^{k}}=\infty \quad \text { for } 0<\lambda<\mu .
$$

Proof. By Theorem 2 we have

$$
A x=\sum_{i=1}^{\infty} \lambda_{i}\left(x, e_{i}\right) e_{i},\left(x, e_{i}\right) \neq 0 \quad \text { for } i=1,2, \cdots,
$$

from which it follows that for any $\lambda_{i} \neq 0$ 


$$
\frac{\left\|A^{k} x\right\|^{2}}{\lambda^{2 k}}=\frac{\left(A^{k} x, A^{k} x\right)}{\lambda^{2 k}}=\frac{\left(A^{2 k} x, x\right)}{\lambda^{2 k}}=\sum_{i=1}^{\infty}\left(\frac{\lambda_{i}}{\lambda}\right)^{2 k}\left|\left(x, e_{i}\right)\right|^{2} .
$$

Now let $\lambda>\lambda_{i}$, so that $0<\left(\lambda_{i} / \lambda\right)<1$ for $i=1,2, \cdots$. Given an arbitrary number $\epsilon>0$ there exists a number $N(\epsilon)$ such that

$$
\sum_{i=N+1}^{\infty}\left|\left(x, e_{i}\right)\right|^{2}<\frac{\epsilon}{2} .
$$

Obviously, since $0<\left(\lambda_{i} / \lambda\right)<1$,

$$
\sum_{i=N+1}^{\infty}\left(\frac{\lambda_{i}}{\lambda}\right)^{2 k}\left|\left(x, e_{i}\right)\right|^{2}<\frac{\epsilon}{2} \quad \text { for } k=1,2, \cdots
$$

and

$$
\sum_{i=1}^{N}\left(\frac{\lambda_{i}}{\lambda}\right)^{2 k}\left|\left(x, e_{i}\right)\right|^{2}<\frac{\epsilon}{2} \quad \text { for } k>k_{0}(\epsilon) \geqq N(\epsilon) .
$$

It follows from (5), (6), and (7) that

$$
\frac{\left\|A^{k} x\right\|^{2}}{\lambda^{2 k}}<\epsilon
$$

for $k>k_{0}(\epsilon)$;

so that $\lim _{k \rightarrow \infty}\left(\left\|A^{k} x\right\| / \lambda^{k}\right)=0$. Observing that by Theorem $3 \lambda_{i}<\mu$ when $\mu$ does not contribute to $x$ the first two assertions of Theorem 4 follow.

Now let $\mu$ contribute to $x$ and for the sake of simplicity, let

$$
\mu=\lambda_{1}=\lambda_{2}=\cdots=\lambda_{n}, \mu>\lambda_{i}
$$

for $i>n$.

By (5) we have

$$
\frac{\left\|A^{k} x\right\|^{2}}{\mu^{2 k}}=\sum_{i=1}^{n}\left|\left(x, e_{i}\right)\right|^{2}+\sum_{i=n+1}^{\infty}\left(\frac{\lambda_{i}}{\mu}\right)^{2 k}\left|\left(x, e_{i}\right)\right|^{2} .
$$

By the same argument as above it is seen that the last term of this equation tends to zero as $k$ tends to infinity so that

$$
\lim _{k \rightarrow \infty} \frac{\left\|A^{k} x\right\|}{\mu^{k}}=\left(\sum_{i=1}^{n}\left|\left(x, e_{i}\right)\right|^{2}\right)^{1 / 2}=\rho>0
$$

which proves the third assertion of Theorem 4 .

If, finally, $0<\lambda<\mu$, there exists by Theorem 3 a characteristic value, say $\lambda_{1}$, such that $\lambda<\lambda_{1}$, and since by (5)

$$
\frac{\left\|A^{k} x\right\|}{\lambda^{k}} \geqq\left(\frac{\lambda_{1}}{\lambda}\right)^{k}\left|\left(x, e_{1}\right)\right|,
$$


the last assertion of our theorem follows readily $\left({ }^{4}\right)$.

We see by Theorem 4 that $\mu$ can be characterized as the greatest lower bound of all real $\lambda$ for which $\lim _{k \rightarrow \infty}\left(\left\|A^{k} x\right\| / \lambda^{k}\right)=0$.

The proof of Theorem 3 depends essentially on the fact that for $|\zeta|<1 / \mu$ the quantities $\left|\zeta \lambda_{i}\right| /\left(1-\left|\zeta \lambda_{i}\right|\right)$ have a finite upper bound. This no longer needs to be true when $\zeta$ equals $1 / \mu$. In this case Theorem 3 does not provide any information about the series (4). But by a closer inspection of the operator $A$ we can prove the following theorem relating the convergence of the series (4) for $\zeta=1 / \mu$ to the contribution of $\mu$ to $x$.

Theorem 5. Let $A x \neq 0$ and $\mu=\lim _{k \rightarrow \infty}\left(\left\|A^{k} x\right\| /\left\|A^{k-1} x\right\|\right)$. Then the series

$$
\sum_{k=1}^{\infty} \frac{\left(A^{k} x, x\right)}{\mu^{k}}
$$

converges if and only if $\mu$ does not contribute to $x$.

We first take up the easier part of the proof. If the series (9) converges then the sequence $\left(A^{k} x, x\right) / \mu^{k}$ tends to zero as $k \rightarrow \infty$ and so does the sequence $\left(A^{2 k} x, x\right) / \mu^{2 k}=\left(\left\|A^{k}\right\| / \mu^{k}\right)^{2}$. Therefore by Theorem 4 we can infer that $\mu$ does not contribute to $x$.

Now let the real number $\lambda_{0} \neq 0$ be in the resolvent set or the continuous spectrum of $A$ and let $\bar{X}$ be the closure of $X$. The adjoint transformation $A^{*}$ is a self-adjoint extension of $A$, defined on $\bar{X}$, and $\lambda$ is in the resolvent set or the continuous spectrum of $A^{*}\left[1\right.$, Paragraph 5]. Therefore $\left(A^{*}-\lambda_{0} I\right)^{-1}$ exists and, by the definition of $A, X$ lies in the domain of $\left(A^{*}-\lambda_{0} I\right)^{-1}$. By $[3$, pp. 342 and 346$]$ we see that

$$
\int_{-\infty}^{\infty}\left(\frac{1}{\lambda-\lambda_{0}}\right)^{2} d\left(E_{\lambda} x, x\right)
$$

exists for every $x$ in $X$, where $E_{\lambda}$ is the resolution of the identity corresponding to $A^{*}$. Since the system of characteristic elements of $A^{*}$ is complete in $\bar{X}$ and since the characteristic manifolds of $A^{*}$ and $A$ which correspond to the same characteristic value $\lambda \neq 0$ coincide [1, Paragraph 5], it follows that the series

$$
\sum_{i=1}^{\infty}\left(\frac{1}{\lambda_{i}-\lambda_{0}}\right)^{2}\left|\left(x, e_{i}\right)\right|^{2}
$$

converges for every $x$ in $X ;\left\{e_{k}\right\}$ is the sequence of elements of $E$ for which $(e, x) \neq 0$.

Now let $\lambda_{0} \neq 0$ be a characteristic value of $A$ which does not contribute to $x$. It follows that $x$ is orthogonal to the characteristic manifold $N\left(A-\lambda_{0} I\right)$. Since by the definition of $A, X$ is the direct sum

(4) An analogous theorem for arbitrary symmetric operators is proved in [4]. 


$$
X=N\left(A-\lambda_{0} I\right)+R\left(A-\lambda_{0} I\right)
$$

we infer that $x$, as well as all the characteristic elements corresponding to characteristic values $\lambda \neq \lambda_{0}$, lies in the linear system $R\left(A-\lambda_{0} I\right)$. Now $A$ is a symmetric finite operator $A^{\prime}$ on $R\left(A-\lambda_{0} I\right)$ [1, Paragraph 2] and by Theorem 1 $\lambda_{0}$ is in the resolvent set or the continuous spectrum of $A^{\prime}$. Since each characteristic value $\lambda \neq \lambda_{0}$ of $A$ is a characteristic value of $A^{\prime}$ and vice versa and since the corresponding characteristic manifolds are equal, it follows that the series (10) converges for each $x$ orthogonal to $N\left(A-\lambda_{0} I\right)$. We may summarize these results by stating that the series (10) converges for any $x$ to which $\lambda_{0} \neq 0$ does not contribute.

If $\lambda_{0} \neq 0$ does not contribute to $x$, then $\lambda_{0}$ does not contribute to $A x$ either. Therefore, the series

$$
\sum_{i=1}^{\infty}\left(\frac{1}{\lambda_{i}-\lambda_{0}}\right)^{2}\left|\left(A x, e_{i}\right)\right|^{2}=\sum_{i=1}^{\infty}\left(\frac{\lambda_{i}}{\lambda_{i}-\lambda_{0}}\right)^{2}\left|\left(x, e_{i}\right)\right|^{2}
$$

is convergent. It follows that the series

$$
\sum_{i=1}^{\infty} \frac{\lambda_{i}}{\lambda_{i}-\lambda_{0}}\left|\left(x, e_{i}\right)\right|^{2}
$$

is also convergent. Suppose now that $\lambda_{0}=\mu$ and that $\mu$ does not contribute to $x$. By Theorem 3 we have $\lambda_{i}<\mu$ for all characteristic values $\lambda_{i}$ contributing to $x$, therefore the series (11) converges absolutely for $\lambda_{0}=\mu$ and we have

$$
\sum_{i=1}^{\infty} \frac{\lambda_{i}}{\lambda_{i}-\mu}\left|\left(x, e_{i}\right)\right|^{2}=-\sum_{i=1}^{\infty} \sum_{k=1}^{\infty}\left(\frac{\lambda_{i}}{\mu}\right)^{k}\left|\left(x, e_{i}\right)\right|^{2} .
$$

By rearranging this series and by using the identity (3) we see that the series (9) converges.

This completes the proof.

The following theorem shows that the usual iteration method $[2 ; 3$, p. $237 ; 5$ ] for determining characteristic values and characteristic elements can be successfully applied if the iteration sequence $A^{k} x /\left\|A^{k} x\right\|$ converges.

Theorem 6. Let $A x$ be different from zero. The sequence $A^{k} x /\left\|A^{k} x\right\|$ converges to an element $h \in X$ if and only if $\mu=\lim _{k \rightarrow \infty}\left(\left\|A^{k} x\right\| /\left\|A^{k-1} x\right\|\right)$ contributes to $x$. In this case $h$ is a normed characteristic element corresponding to the characteristic value $\mu$. If $\mu$ does not contribute to $x$, then $A^{k} x /\left\|A^{k} x\right\|$ converges weakly to zero.

Proof. Let $\mu$ contribute to $x$ and for the sake of simplicity, let $\mu=\lambda_{1}$ $=\lambda_{2}=\cdots=\lambda_{n}, \mu>\lambda_{i}$ for $i>n$, in the expansion

$$
A x=\sum_{i=1}^{\infty} \lambda_{i}\left(x, e_{i}\right) e_{i}
$$


We then have

$$
\frac{A^{k} x}{\left\|A^{k} x\right\|}=\frac{A^{k} x}{\mu^{k}} \cdot \frac{\mu^{k}}{\left\|A^{k} x\right\|}=\frac{\mu^{k}}{\left\|A^{k} x\right\|}\left[\sum_{i=1}^{n}\left(x, e_{i}\right) e_{i}+\sum_{i=n+1}^{\infty}\left(\frac{\lambda_{i}}{\mu}\right)^{k}\left(x, e_{i}\right) e_{i}\right] .
$$

Now as $k \rightarrow \infty$ the sequence $\sum_{i=n+1}^{\infty}\left(\lambda_{i} / \mu\right)^{k}\left(x, e_{i}\right) e_{i}$ tends to zero (see the proof of Theorem 4) and the sequence $\mu^{k} /\left\|A^{k} x\right\|$ tends to $\left(\sum_{i=1}^{n}\left|\left(x, e_{i}\right)\right|^{2}\right)^{-1 / 2}$ by Theorem 4 . Therefore $A^{k} x /\left\|A^{k} x\right\|$ converges to a normed characteristic element corresponding to $\mu$.

Now we suppose that $\mu$ does not contribute to $x$. Then all the characteristic values $\lambda_{i}$ in (11) are less than $\mu$ by virtue of Theorem 3 . If $e$ is an element of $E$, we have

$$
\left(\frac{A^{k} x}{\left\|A^{k} x\right\|}, e\right)=\frac{1}{\left\|A^{k} x\right\|}\left(\sum_{i=1}^{\infty} \lambda_{i}^{k}\left(x, e_{i}\right) e_{i}, e\right)=\left\{\begin{array}{l}
0 \text { in case } e \neq e_{i}, i=1,2, \cdots, \\
\frac{\lambda_{j}^{k}}{\left\|A^{k} x\right\|}\left(x, e_{j}\right) \text { in case } e=e_{j} .
\end{array}\right.
$$

Since $0<\lambda_{j}<\mu$, the sequence $\lambda_{j}^{k} /\left\|A^{k} x\right\|$ converges to zero as $k \rightarrow \infty$ by Theorem 4 . Therefore we have

$$
\lim _{x \rightarrow \infty}\left(\frac{A^{k} x}{\left\|A^{k} x\right\|}, e\right)=0 \quad \text { for every } e \in E .
$$

Recalling Theorem 2 we infer that $\left(A^{k} x /\left\|A^{k} x\right\|, y\right) \rightarrow 0$ for every $y$ in the closure $Y$ of $A X$. Obviously $\left(A^{k} x /\left\|A^{k} x\right\|, z\right)=0$ for every $z$ in the orthogonal complement of $Y$ in $\bar{X}$, thus it follows that for every element $\bar{x} \in \bar{X}$ $\lim _{k \rightarrow \infty}\left(A^{k} x /\left\|A^{k} x\right\|, \bar{x}\right)=0$. Hence $A^{k} x /\left\|A^{k} x\right\|$ converges weakly to zero. We see by this result, that if $\mu$ does not contribute to $x$ the sequence $A^{k} x /\left\|A^{k} x\right\|$ cannot converge strongly, because otherwise its limit would equal the weak limit 0 which is impossible, since $A^{k} x /\left\|A^{k} x\right\|$ is a normed element.

This completes the proof of Theorem 6 .

Corollary. Suppose $A x \neq 0$. Then $\mu=\lim _{k \rightarrow \infty}\left(\left\|A^{k} x\right\| /\left\|A^{k-1} x\right\|\right)$ contributes to $x$ if and only if there is a positive constant $\alpha$ (which depends only on $x$ but not on $k$ ) such that

$$
\left\|A^{2 k} x\right\| \leqq \alpha\left\|A^{k} x\right\|^{2} \quad \text { for } k=1,2, \cdots .
$$

Proof. Let $\mu$ contribute to $x$. Then by Theorem 6 the sequence $A^{k} x /\left\|A^{k} x\right\|$ converges to a characteristic element $h$ corresponding to $\mu$. Therefore

$$
\lim _{k \rightarrow \infty}\left(\frac{A^{2 k} x}{\left\|A^{2 k} x\right\|}, x\right)=\lim _{k \rightarrow \infty} \frac{1}{\left\|A^{2 k} x\right\|}\left(A^{k} x, A^{k} x\right)=\lim _{k \rightarrow \infty} \frac{\left\|A^{k} x\right\|^{2}}{\left\|A^{2 k} x\right\|}=(h, x) .
$$

$(h, x)$ is different from zero, otherwise $\left(h, A^{k} x /\left\|A^{k} x\right\|\right)=\left(1 /\left\|A^{k} x\right\|\right)\left(A^{k} h, x\right)$ $=\left(\mu^{k} /\left\|A^{k} x\right\|\right)(h, x)$ would equal zero and, by Theorem $4, \lim _{k \rightarrow \infty}\left(h, A^{k} x /\left\|A^{k} x\right\|\right)$ $=(h, h)$ would equal zero contrary to $h \neq 0$. Therefore it follows that the 
sequence $\left\|A^{k} x\right\|^{2} /\left\|A^{2 k} x\right\|$ has a positive lower bound $1 / \alpha$ so that (13) holds. If on the other hand (13) is valid, then $\left(\mathrm{A}^{2 k} x /\left\|A^{2 k} x\right\|, x\right)$, and therefore $\left(A^{k} x /\left\|A^{k} x\right\|, x\right)$ cannot converge to zero. Hence, by Theorem $6, \mu$ contributes to $x$.

For the rest of this paper we need a concept first introduced by Wavre [4]. We call a bounded symmetric operator $B$ regular, if for each element $x$ of its domain with $B x \neq 0, \lim _{k \rightarrow \infty}\left(\left\|B^{k} x\right\| / \mu_{x}^{k}\right)$ is different from zero (where $\left.\mu_{x}=\lim _{k \rightarrow \infty}\left(\left\|B^{k} x\right\| /\left\|B^{k-1} x\right\|\right)\right)\left({ }^{5}\right)$.

THEOREM 7. $A$ is regular if and only if for each $x \in X$ with $A x \neq 0$ the characteristic values contributing to $x$ can be arranged in a nonincreasing sequence.

Proof. Suppose first that the characteristic values $\lambda_{i}$ contributing to $x$ can be arranged in a nonincreasing sequence $\lambda_{1}=\lambda_{2}=\cdots=\lambda_{n}>\lambda_{n+1} \geqq \cdots \cdot$ Then $\sup \lambda_{i}=\lambda_{1}$, so that the least upper bound of the $\lambda_{i}$ 's contributes to $x$. By Theorems 3 and 4 we have therefore $\lim _{k \rightarrow \infty}\left(\left\|A^{k} x\right\| / \mu_{x}^{k}\right) \neq 0$, hence $A$ is regular.

Now let $A$ be regular and $A x \neq 0$. Then with $\mu=\mu_{x}$ we have by Theorems 3 and 4

$$
A x=\sum_{i=1}^{n} \mu\left(x, e_{i}\right) e_{i}+\sum_{i=n+1}^{\infty} \lambda_{i}\left(x, e_{i}\right) e_{i}, \lambda_{i}<\mu \quad \text { for } i=n+1, n+2, \cdots .
$$

Since, by the definition of $A, x=e+f, e \in N(A-\mu I), f \in R(A-\mu I)$, it follows that

$$
A e-\sum_{i=1}^{n} \mu\left(x, e_{i}\right) e_{i}=\sum_{i=n+1}^{\infty} \lambda_{i}\left(x, e_{i}\right) e_{i}-A f .
$$

The first term of this equation is an element of $N(A-\mu I)$, the second term is an element of $R(A-\mu I)$, but because these two linear systems are orthogonal to each other both terms must vanish. Therefore

$$
A f=\sum_{i=n+1}^{\infty} \lambda_{i}\left(e+f, e_{i}\right) e_{i}=\sum_{i=n+1}^{\infty} \lambda_{i}\left(f, e_{i}\right) e_{i},
$$

since $\left(e, e_{i}\right)=0$ for $i \geqq n+1 . \mu_{f}=\sup _{i \geq n+1} \lambda_{i}$ is equal to one of the $\lambda_{i}$ 's, $i \geqq n+1$, because $A$ is regular, and therefore $\mu_{f}<\mu_{x}$. The proof can now be finished by mathematical induction.

Next we consider the relation between the regularity of $A^{*}$ and the spectrum of $A$. In order to state Theorem 8 it is convenient to introduce the following definition.

We say that $A$ has a band spectrum if every limit point $\lambda_{0} \neq 0$ of characteristic values of $A$ can be approximated only by characteristic values greater

(b) The existence of these limits is proved in [4]. 
than $\lambda_{0}$ (so that in a left-hand neighborhood $\lambda_{0}-\epsilon<\lambda<\lambda_{0}$ there are no characteristic values).

It follows immediately that the number of these limit points is at most enumerable.

TheOREM 8. $A$ has a band spectrum if and only if $A^{*}$ is regular.

Proof. Let $A^{*}$ be regular and let $\lambda_{0} \neq 0$ be a limit point of characteristic values of $A^{*}$. Suppose there is a sequence of different characteristic values $\lambda_{i} \neq 0$ of $A^{*}, i=1,2, \cdots$, with $\lambda_{i}<\lambda_{0}, \lambda_{i} \rightarrow \lambda_{0}$ for $i \rightarrow \infty$. Let $\alpha_{1}, \alpha_{2}, \cdots$, be an arbitrary sequence of complex numbers with $\sum_{i=1}^{\infty}\left|\alpha_{i}\right|^{2}<+\infty, \alpha_{i} \neq 0$, and consider the element $\bar{x}=\sum_{i=1}^{\infty} \alpha_{i} e_{i}$ of $\bar{X}$, where $e_{i}$ is a normed characteristic element corresponding to $\lambda_{i}$. Since $\lambda_{i} \alpha_{i} \neq 0$ we have

$$
A^{*} \bar{x}=\sum_{i=1}^{\infty} \lambda_{i} \alpha_{i} e_{i} \neq 0
$$

The proofs of the Theorems 3 and 4 were based only on the expansion (1) and the positiveness of $A$. Here we have the expansion (14), and the positiveness of $A^{*}$ follows readily from the positiveness of $A$. Hence both theorems hold for $A^{*} \bar{x}$ and it results that

$$
\lim _{k \rightarrow \infty} \frac{\left\|\left(A^{*}\right)^{k} \bar{x}\right\|}{\left\|\left(A^{*}\right)^{k-1} \bar{x}\right\|}=\sup \lambda_{i}=\lambda_{0}, \quad \lim _{x \rightarrow \infty} \frac{\left\|\left(A^{*}\right)^{k} \bar{x}\right\|}{\lambda_{0}^{k}}=0 .
$$

But the last equation contradicts the regularity of $A^{*}$. Hence $\lambda_{0}$ cannot be approximated by characteristic values less than $\lambda_{0}$. Therefore $A^{*}$ has a band spectrum. Since the characteristic values $\neq 0$ of $A$ and $A^{*}$ are the same by [1, Paragraph 5], it follows that $A$ has also a band spectrum.

Now suppose that $A$ has a band spectrum and let $\bar{x}$ be an arbitrary element in $\bar{X}$ with $A^{*} \bar{x} \neq 0$. Then the expansion

$$
A^{*} \bar{x}=\sum_{i=1}^{\infty} \lambda_{i}\left(\bar{x}, e_{i}\right) e_{i}
$$

is valid, where the $e_{i}$ 's are in $E$ and the $\lambda_{i}$ 's are the corresponding characteristic values of $A$ (see [1, Paragraph 5]). By Theorem 3 we infer from this expansion that $\mu_{\bar{x}}=\lim _{k \rightarrow \infty}\left(\left\|\left(A^{*}\right)^{k} \bar{x}\right\| /\left\|\left(A^{*}\right)^{k-1} \bar{x}\right\|\right)$ equals sup $\lambda_{i}$. But since $A$ has a band spectrum, sup $\lambda_{i}$ is one of the characteristic values $\lambda_{1}, \lambda_{2}, \cdots$, hence, by Theorem $4, \lim _{k \rightarrow \infty}\left(\left\|\left(A^{*}\right)^{k} \bar{x}\right\| / \mu_{x}^{k}\right) \neq 0$, i.e. $A^{*}$ is regular.

The regularity of $A$ does not imply that $A$ has a band spectrum. Consider for example the space $X$ of all sequences $\left\{\alpha_{1}, \alpha_{2}, \cdots\right\}$ of complex numbers where only finitely many $\alpha_{i}$ are different from zero and define the linear opertions and the inner product in the usual way. Define an operator $A$ on $X$ by the diagonal matrix 


$$
\left(\begin{array}{llll}
\lambda_{1} & & & 0 \\
& \lambda_{2} & \\
& & & \\
0 & & & .
\end{array}\right)
$$

$\lambda_{n}=1-1 / n . A$ is obviously symmetric, positive, finite and regular but has no band spectrum because the characteristic values $\lambda_{n}$ approximate their limit point 1 from the left side.

Suppose now that the operator $A$ on $X$ is finite, symmetric and bounded, but not necessarily positive. Then $A^{2}$ is finite by [1, Paragraph 2] and obviously symmetric, bounded and positive. Thus all our theorems can be applied to $A^{2}$ and we can deduce from them, in the conventional way, the corresponding theorems for $A$. Without going into detail we state only the following theorem.

THEOREM 9. Let $A$ be a finite, symmetric and bounded operator on $X$ and $A x \neq 0$. Then the sequence $A^{2 k} /\left\|A^{2 k} x\right\|$ converges to an element $h \in X$ if and only if $\mu^{1 / 2}$ or $-\mu^{1 / 2}$ is a characteristic value of $A$ contributing to $x$, where $\mu=\lim _{k \rightarrow \infty}\left(\left\|A^{2 k} x\right\| /\left\|A^{2 k-2} x\right\|\right)$. If $h$ exists, at least one of the elements

$$
e^{\prime}=h+\left(1 / \mu^{1 / 2}\right) A h, e^{\prime \prime}=h-\left(1 / \mu^{1 / 2}\right) A h
$$

is a characteristic element of $A$ corresponding to the characteristic value $\mu^{1 / 2}$ or $-\mu^{1 / 2}$ respectively.

This theorem follows immediately from Theorems 2 and 6 , since $A e^{\prime}$ $=\mu^{1 / 2} e^{\prime}, A e^{\prime \prime}=-\mu^{1 / 2} e^{\prime \prime}$ and at least one of the elements $e^{\prime}, e^{\prime \prime}$ is different from zero, because $e^{\prime}+e^{\prime \prime}=h$ and $h \neq 0$.

\section{REFERENCES}

1. H. Heuser, Über Operatoren mit endlichen Defekten, Dissertation, Tübingen, 1956.

2. O. D. Kellogg, On the existence and closure of sets of characteristic functions, Math. Ann. vol. 86 (1922) pp. 14-17.

3. F. Riesz and B. Sz.-Nagy, Leçons d'analyse fonctionnelle, Budapest, 1952.

4. R. Wavre, L'iteration directe des opérateurs hermitiens et deux théries qui en dépendent, Comment. Math. Helv. vol. 15 (1943) pp. 299-317.

5. H. Wielandt, Das Iterationsverfahren bei nicht selbstadjungierten linearen Eigenwertaufgaben, Math. Z. vol. 50 (1944) pp. 93-143.

Range Instrumentation Development Division, White Sands Missile Range, New Mexico 